\title{
Inatividade física e sintomas de depressão, ansiedade e estresse em adolescentes estudantes
} Physical inactivity and symptoms of depression, anxiety and stress in adolescent students Inactividad física y síntomas de depresión, ansiedad y estrés en estudiantes adolescentes

\author{
Marcos Paulo da Silva Costa ${ }^{1}$ (D) https://orcid.org/0000-0002-8934-7056 \\ Ademir Schmidt' 1 ib https://orcid:org/0000-0003-4171-5101 \\ Priscila Valverde de Oliveira Vitorino ${ }^{1}$ in https://orcid.org/0000-0002-5487-4649 \\ Krislainy de Sousa Corrêa ${ }^{1}$ [
}

Como citar:

Costa MP, Schmidt A, Vitorino PV, Corrêa KS. Inatividade física e sintomas de depressão, ansiedade e estresse em adolescentes estudantes. Acta Paul Enferm. 2021;34:eAPE03364.

DOI

http://dx.doi.org/10.37689/actaape/2021A003364

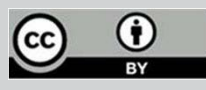

Descritores

Adolescência; Sedentarismo; Atividade física; Depressão; Ansiedade; Estresse

Keywords

Adolescence; Sedentary lifestyle; Physical activity; Depression; Anxiety; Stress

Descriptores

Adolescencia; Estilo de vida sedentario; Actividad física; Depresión; Ansiedad; Estrés

Submetido

10 de Janeiro de 2020

Aceito

2 de Dezembro de 2020

Autor correspondente

Marcos Paulo da Silva Costa E-mail:mpsc.ead@gmail.com

\section{Resumo}

Objetivo: Comparar a prática de atividade física habitual e sintomas de depressão, ansiedade e estresse entre estudantes adolescentes de escolas públicas de tempo integral e parcial.

Métodos: Estudo transversal, analítico, com amostragem estratificada proporcional por conglomerados, realizado em Goiânia, GO, em 2018. A amostra incluiu 516 estudantes adolescentes, sendo 277 de escola de tempo parcial e 239 de escola de tempo integral. Para avaliar o nível de atividade física, foi aplicado 0 International Physical Activity Questionnaire. 0 rastreio de sintomas de depressão, ansiedade e estresse foi avaliado pela Escala de Depressão, Ansiedade e Estresse para Adolescentes. Foi utilizado para avaliar as variáveis categóricas o Teste Qui-Quadrado ou Teste Exato de Fisher. Adotou-se o General Linear Model (GLM) univariado para comparação entre as variáveis contínuas. A correção da homogeneidade foi realizada pelo Teste de Levene. 0 nível de significância adotado foi de $5 \%$.

Resultados: A média de idade foi de 15,95 $\pm 1,15$ anos. A prevalência de adolescentes estudantes do tempo parcial que relataram ser independentes e responsáveis parcialmente pelas despesas foi de $13,4 \%(p<0,005)$. A prevalência geral de sedentarismo foi de $93,5 \%$. A frequência de alunos de tempo integral que afirmaram que a vida não tinha sentido foi de $13 \%(p<0,05)$. Os alunos das escolas de tempo integral apresentaram menor frequência de atividade física moderada a vigorosa quando comparados aos de tempo parcial $(4,10$ $\pm 3,60$ versus $4,80 \pm 3,70$ dias por semana) $(p<0,05)$.

Conclusão: Os estudantes adolescentes do sistema público de ensino demonstraram alto índice de inatividade física, com maior proporção de inativos nas escolas de período integral. Além disso, esses estudantes vivenciam aspectos negativos de ansiedade, estresse e depressão.

\section{Abstract}

Objective: To compare habitual physical activity and symptoms of depression, anxiety and stress among adolescent students of full-time and part-time public schools.

Methods: A cross-sectional analytical study with stratified proportional sampling by clusters, conducted in Goiânia, GO, in 2018. The sample included 516 adolescent students, 277 from part-time school and 239 from full-time school. To assess the level of physical activity, the International Physical Activity Questionnaire was applied. Screening for symptoms of depression, anxiety and stress was assessed by the Depression Anxiety Stress Scale. The chi-square test or Fisher's exact test was used to assess the categorical variables. The univariate General Linear Model (GLM) was adopted to compare continuous variables. Homogeneity correction was performed using the Levene test. The level of significance adopted was $5 \%$. 
Results: The mean age was $15.95 \pm 1.15$ years old. The prevalence of adolescent part-time students who reported being independent and partially responsible for expenses was $13.4 \%(p<0.005)$. The general prevalence of physical inactivity was $93.5 \%$. The frequency of full-time students who stated that life was meaningless was $13 \%(p<0.05)$. Students in full-time schools had a lower frequency of moderate to vigorous physical activity when compared to part-time students $(4.10 \pm 3.60$ versus $4.80 \pm 3.70$ days a week $(p<0.05)$.

Conclusion: Adolescent students in the public school system showed a high rate of physical inactivity, with a higher proportion of inactive in full-time schools. Moreover, these students experience negative aspects of anxiety, stress and depression.

\section{Resumen}

Objetivo: Comparar la práctica de actividad física habitual y síntomas de depresión, ansiedad y estrés entre estudiantes adolescentes de escuelas públicas de jornada completa y simple.

Métodos: Estudio transversal, analítico, con muestreo estratificado proporcional por grupos, realizado en Goiânia, estado de Goiás, en 2018. La muestra incluyó 516 estudiantes adolescentes, de los cuales 277 asistían a la escuela en jornada simple y 239 en jornada completa. Para evaluar el nivel de actividad física, se aplicó el International Physical Activity Questionnaire. La investigación de síntomas de depresión, ansiedad y estrés fue analizada mediante la Escala de Depresión, Ansiedad y Estrés para Adolescentes. Para evaluar las variables categóricas, se utilizó la prueba $\chi^{2}$ de Pearson 0 la prueba exacta de Fisher. El General Linear Model (GLM) univariado fue utilizado para la comparación entre las variables continuas. La corrección de la homogeneidad fue realizada mediante la prueba de Levene. El nivel de significación adoptado fue del $5 \%$.

Resultados: El promedio de edad fue de 15,95 $\pm 1,15$ años. La prevalencia de estudiantes adolescentes en jornada simple que relataron ser independientes y parcialmente responsables por los gastos fue del 13,4 \% ( $<<0,005)$. La prevalencia general del sedentarismo fue del 93,5\%. La frecuencia de alumnos en jornada completa que afirmaron que la vida no tenía sentido fue del $13 \%(p<0,05)$. Los alumnos de las escuelas de jornada completa presentaron menor frecuencia de actividad física moderada a fuerte en comparación con los de jornada simple $(4,10 \pm 3,60$ versus 4,80 $\pm 3,70$ días por semana) $(p<0,05)$.

Conclusión: Los estudiantes adolescentes del sistema público de educación demostraron un alto índice de inactividad física, con una mayor proporción de inactivos en las escuelas de jornada completa. Además, estos estudiantes sufren aspectos negativos de ansiedad, estrés y depresión.

\section{Introdução}

A prática de atividade física é definida como qualquer movimento corporal produzido pelos músculos, que exige dispêndio de energia acima dos níveis de repouso, ${ }^{(1)}$ particularmente, na adolescência. Beneficia a vida cotidiana, como melhora na capacidade física e auto estima, estímulo de socialização e integração no seu grupo social e maior empenho na busca por objetivos de vida. ${ }^{(2)}$ Embora os benefícios da prática de atividade física para adolescentes já estejam bem estabelecidos, a prevalência de inatividade física no Brasil é de $85,2 \%{ }^{(3)}$ e no mundo de $81 \%{ }^{(4)}$ utilizando diferentes instrumentos de medida.

O comportamento sedentário atinge cerca de $60 \%$ dos adolescentes, ${ }^{(3,5)}$ e tem sido evidenciado como um risco para várias alteraçóes orgânicas durante o período da adolescência, como doenças crônicas não transmissíveis, a exemplo da obesidade, diabetes mellitus e doenças cardiovasculares. ${ }^{(6)} \mathrm{O}$ estilo de vida ocioso dessa populaçáo vem aumentando com uso e auxílio cada vez maior das tecnologias (computador, smartphones, tablets), reduzindo os momentos de lazer com práticas de atividades físicas de forma significativa. ${ }^{(7)}$

O comportamento sedentário em estudantes pode potencializar a experimentaçáo de aspec- tos negativos de depressão, ansiedade e estresse. Adolescentes são vulneráveis a situações estressoras e podem relatar sintomas nocivos, levando-os a um prejuízo tanto na parte física como mental. ${ }^{(8)} \mathrm{Uma}$ metanálise identificou que o comportamento sedentário foi associado a sintomas de depressão em crianças e adolescentes. ${ }^{(9)}$

Embora existam estudos que identificaram que a atividade física é fator de proteção em adultos para depressão, ${ }^{(10)}$ ansiedade ${ }^{(11)} \mathrm{e}$ estresse, ${ }^{(12)}$ verifica-se escassez de trabalhos com referência à atividade física e sintomas de depressão, ansiedade e estresse em adolescentes.

Desta forma, o estudo teve por objetivo comparar a prática de atividade física habitual e sintomas de depressáo, ansiedade e estresse entre estudantes adolescentes de escolas públicas estaduais de tempo integral e de tempo parcial no município de Goiânia.

\section{Métodos}

Estudo transversal, analítico, com amostragem estratificada proporcional por conglomerados, realizado de fevereiro a julho de 2018, com alunos entre quatorze e dezenove anos, de ambos os sexos, matriculados em escolas públicas de ensino médio de pe- 
ríodo integral e parcial de Goiânia, estado de Goiás. A capital é considerada uma metrópole, localizada na regiáo central do Brasil, apresentando um total de 34.704 adolescentes matriculados em dezembro de 2017. Para o cálculo da amostra, foi estimado um total de 516 estudantes. Desses, 277 eram de escola de tempo parcial, e 239 eram de escola de tempo integral, considerando um tamanho de efeito de 0,30 , com poder observado e esperado de $80 \%$ e $\alpha=0,05$, incluindo $10 \%$ de perdas. Dezoito das 75 escolas foram sorteadas para representar todas as regióes de Goiânia.

Para efeito deste estudo e baseado na divisão distrital, o município de Goiânia foi dividido em 9 regiôes: Noroeste, Sudoeste, Mendanha, Vale do Meia Ponte, Norte, Sul, Leste, Oeste e Central. Os critérios de inclusão foram: alunos matriculados na rede de ensino estadual do Município de Goiânia; nas modalidades de tempo parcial e integral; de ambos os sexos; alunos regulares da modalidade de tempo parcial ou integral por, no mínimo. dois meses antes da entrada no estudo; e idade entre 14 e 19 anos. Os critérios de exclusão foram: regióes que não possuíam as modalidades de tempo parcial e/ou integral; alunos com deficiência física, como paraplegia, hemiplegia; alunos que os responsáveis não assinaram o Termo de Consentimento Livre e Esclarecido (TCLE); e alunos que não assinassem o Termo de Assentimento Informado Livre e Esclarecido (TAILE).

Para a caracterização do perfil sociodemográfico dos escolares, foi utilizada uma ficha semiestruturada composta por informaçóes referentes à idade, sexo, situação habitacional, tipo de habitação, orçamento familiar, renda familiar mensal em salário mínimo (SM) de referência no Censo Demográfico 2018 do Instituto Brasileiro de Geografia e Estatística (IBGE) (R\$ 954,00), hábitos de vida como tabagismo, alcoolismo e uso de drogas ilícitas.

Para a avaliação do nível de atividade física dos adolescentes estudantes, foi utilizada a versão curta do Questionário Internacional de Atividade Física (IPAQ), validado no Brasil, composto por oito questões abertas. As informaçóes do IPAQ estimam o tempo gasto, por semana, em várias dimensóes de atividade física (caminhada, atividade física de intensidade moderada e vigorosa) e de comportamento sedentário (posição sentada). A classificação do IPAQ registra a frequência (dias) e duração (minutos) em que os alunos praticam atividade física, podendo ser classificados em muito ativo, ativo, irregularmente ativo e sedentário. ${ }^{(13)}$

Utilizando o levantamento feito pelo IPAQ sobre o nível de atividade física dos adolescentes, foram considerados os critérios da classificação da OMS em fisicamente ativo (adolescentes que realizam, pelo menos, 300 minutos semanais de atividade física moderada a vigorosa) ou fisicamente inativo (adolescentes que realizam atividade física, entretanto de forma insuficiente para serem classificados como ativos, pois não cumprem as recomendações da OMS de 300 minutos semanais de atividade física). ${ }^{(1)} \mathrm{O}$ comportamento sedentário desses adolescentes estudantes foi avaliado por meio do tempo médio em minutos sentado em dia da semana e em dia de final de semana.Os adolescentes não devem passar mais que duas horas por dia em comportamento sedentário, ${ }^{(14)}$ o que corresponde a dez horas durante a semana e quatro horas no fim de semana (total de 14 horas semanais).

A partir da Escala de Depressão, Ansiedade e Estresse para Adolescentes (EDAE-A), foi possível rastrear os sintomas de depressão, ansiedade e estresse dos participantes da pesquisa. Essa escala foi adaptada do DASS-21 (Depression Anxiety Stress Scale), sendo validada no Brasil, composta por 21 questôes. As pontuaçóes dos domínios depressão, ansiedade e estresse são determinadas pela soma dos escores dos 21 itens. $^{(8)}$ Para sua interpretação, considerou-se o somatório de pontos correspondentes às respostas: 0 (não aconteceu comigo essa semana), 1 (aconteceu comigo algumas vezes na semana), 2(aconteceu comigo em boa parte da semana), 3 (aconteceu comigo na maior parte do tempo da semana).

Os níveis de atividade física e sintomas de depressão, ansiedade e estresse foram comparados entre os estudantes de escola de tempo parcial e integral. Os dados de distribuição não paramétrica foram padronizados pelo escore Z. O Teste Qui-Quadrado/ Teste de Fisher foi utilizado para avaliar as variáveis categóricas, e foi adotado o General Linear Model 
(GLM) univariado para comparação entre as variáveis contínuas. As frequências e parâmetros de nível de atividade física e sedentarismo foram determinadas para o grupo geral, e foram estratificadas por modalidades de tempo parcial e integral. O nível de significância adotado foi de $5 \%(\mathrm{p}<0,05)$.

O estudo foi aprovado pelo Comitê de Ética em Pesquisa da Pontifícia Universidade Católica de Goiás (CAAE 74378817.9.0000.0037), conforme orientaçôes para a pesquisa com seres humanos constantes na Resolução 466/2012 do Conselho Nacional de Saúde.

\section{Resultados}

A média da idade dos estudantes que participaram do estudo foi de 15,95 $\pm 1,15$ anos. Dos 516 alunos pesquisados, 242(46,9\%) afirmaram que habitavam em imóvel próprio; $38(7,4 \%)$ possuíam imóvel próprio financiado; $210(40,7 \%)$ moravam em imóvel alugado; $15(2,9 \%)$ moravam em imóvel cedido; e $11(2,1 \%)$ habitavam em imóvel herdado por parentes. Em relação à situação familiar dos adolescentes, $272(52,7 \%)$ responderam que os pais eram casados; $207(40,1 \%)$ tinham pais separados; $17(3,3 \%)$ afirmaram que o pai é falecido; e $7(1,4 \%)$ relataram que a mãe é falecida.

Os alunos de escola de período integral e parcial eram semelhantes quanto ao sexo e condiçóes de habitação. No entanto, os alunos de escola de período parcial tinham maior participação na renda familiar, geralmente baixa, conforme demonstrado na tabela 1.

Quanto ao nível de atividade física, a maioria dos adolescentes avaliados eram inativos, totalizando $93,5 \%$ das escolas de tempo parcial e $98,3 \%$ das escolas de tempo integral (Figura 1).

Os estudantes das escolas de tempo integral apresentaram menor frequência de atividade física referentes à caminhada $(\mathrm{p}=0,04)$, somatório total da atividade física $(\mathrm{p}=0,04)$ e somatório das atividades físicas moderadas e vigorosas $(\mathrm{p}=0,03)$ (Tabela 2 ).

O comportamento sedentário dos estudantes adolescentes de ambas as escolas foi evidenciado quando foram demonstrados outros parâmetros da atividade
Tabela 1. Características dos adolescentes matriculados em escolas públicas estaduais e comparação entre os grupos de tempo integral e parcial

\begin{tabular}{|c|c|c|c|c|}
\hline Características & $\begin{array}{l}\text { Total } \\
\mathrm{n}(\%)\end{array}$ & $\begin{array}{c}\text { Período } \\
\text { Integral } \\
\mathrm{n}(\%)\end{array}$ & $\begin{array}{c}\text { Período } \\
\text { Parcial } \\
\mathrm{n}(\%)\end{array}$ & $p$-value \\
\hline \multicolumn{5}{|l|}{$\operatorname{Sexo}(n=516)$} \\
\hline Masculino & $214(41,5)$ & $96(40,2)$ & $118(42,6)$ & 0,57 \\
\hline Feminino & $302(58,5)$ & $143(59,8)$ & $159(57,4)$ & \\
\hline \multicolumn{5}{|l|}{ Escolaridade } \\
\hline $1^{\circ}$ ano & $235(45,6)$ & $104(43,5)$ & $131(47,3)$ & 0,59 \\
\hline $2^{\circ}$ ano & $189(36,6)$ & $93(38,9)$ & $96(34,7)$ & \\
\hline $3^{\circ}$ ano & $92(17,8)$ & $42(17,6)$ & $50(18,1)$ & \\
\hline \multicolumn{5}{|l|}{ Habitação } \\
\hline Com família & $314(60,9)$ & $142(59,4)$ & $172(62,1)$ & 0,14 \\
\hline Sozinho & $4(0,8)$ & $2(0,8)$ & $2(0,7)$ & \\
\hline Com amigos & $6(1,2)$ & $0(0,0)$ & $6(2,2)$ & \\
\hline De favor & $48(9,3)$ & $27(11,3)$ & $21(7,6)$ & \\
\hline Com parentes & $3(0,6)$ & $0(0,0)$ & $3(1,1)$ & \\
\hline Com o pai & $25(4,8)$ & $14(5,9)$ & $11(4,0)$ & \\
\hline Com a mãe & $108(20,9)$ & $50(20,9)$ & $58(20,9)$ & \\
\hline Com os irmãos & $8(1,5)$ & $4(1,7)$ & $4(1,7)$ & \\
\hline \multicolumn{5}{|l|}{ Orçamento da família } \\
\hline Depende dos pais & $414(80,2)$ & $204(85,4)$ & $210(75,85)$ & $0,003^{*}$ \\
\hline Depende dos parentes & $22(4,3)$ & $13(5,4)$ & $9(3,2)$ & \\
\hline Indep. financeiramente & $21(4,1)$ & $8(3,3)$ & $13(4,7)$ & \\
\hline Indep. e resp.parc. pelas desp. & $47(9)$ & $10(4,2)$ & $37(13,4)$ & \\
\hline Indep. e resp.pelas desp. domésticas & $12(2,3)$ & $4(1,7)$ & $8(2,9)$ & \\
\hline \multicolumn{5}{|l|}{ Renda familiar } \\
\hline 1-2 salários & $287(55,6)$ & $131(54,8)$ & $156(56,3)$ & 0,19 \\
\hline 3-4 salários & $185(35,9)$ & $82(34,3)$ & $103(37,2)$ & \\
\hline$>5$ salários & $44(8,5)$ & $26(10,9)$ & $18(6,5)$ & \\
\hline
\end{tabular}

GLM univariado, $\mathrm{p}<0,05^{\star}$; Indep. - independente; resp. - responsável; parc. - parcialmente; desp. - despesas

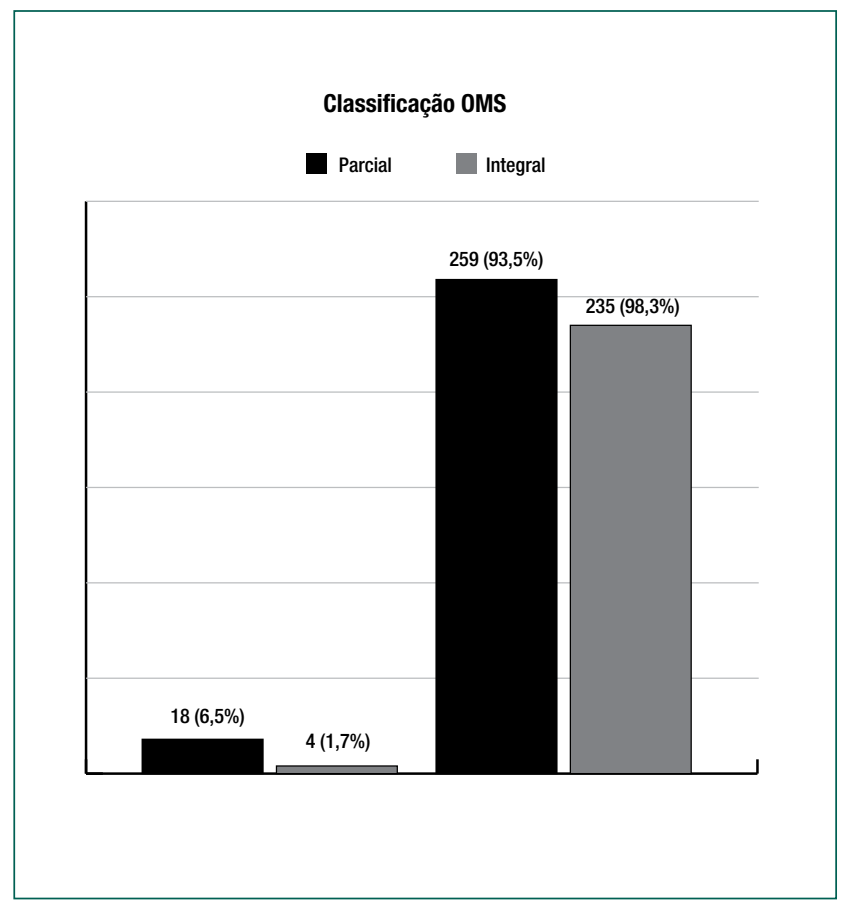

Qui-Quadrado, $\mathrm{p}-0,96$

Figura 1. Proporção da prática de atividade física de adolescentes estudantes de acordo com as diretrizes da OMS 
Tabela 2. Comparação dos parâmetros do nível de atividade física entre os grupos de tempo integral e parcial

\begin{tabular}{|c|c|c|c|c|c|}
\hline Atividade física & Dias/Minutos & $\begin{array}{c}\text { Total } \\
\mathrm{n}=516\end{array}$ & $\begin{array}{c}\text { Integral } \\
\mathrm{n}=239\end{array}$ & $\begin{array}{l}\text { Parcial } \\
n=277\end{array}$ & $p$-value \\
\hline \multirow{2}{*}{ Caminhada } & Frequência semanal (dias) & $3,53 \pm 2,42$ & $3,30 \pm 2,48$ & $3,72 \pm 2,36$ & $0,04^{*}$ \\
\hline & Duração do esforço (min) & $52,71 \pm 48,82$ & $50,88 \pm 49,69$ & $54,29 \pm 48,09$ & 0,43 \\
\hline \multirow[t]{2}{*}{ Moderada } & Frequência semanal (dias) & $2,75 \pm 2,34$ & $2,63 \pm 2,28$ & $2,88 \pm 2,39$ & 0,25 \\
\hline & Duração do esforço (min) & $58,98 \pm 53,73$ & $59,87 \pm 51,64$ & $58,21 \pm 55,56$ & 0,72 \\
\hline \multirow[t]{2}{*}{ Vigorosa } & Frequência semanal (dias) & $1,71 \pm 1,99$ & $1,61 \pm 1,91$ & $1,80 \pm 2,05$ & 0,28 \\
\hline & Duração do esforço (min) & $46,42 \pm 54,21$ & $43,47 \pm 52,37$ & $48,97 \pm 55,71$ & 0,25 \\
\hline \multirow[t]{2}{*}{ Somatório total } & Frequência semanal (dias) & $8,00 \pm 4,91$ & $7,54 \pm 5,00$ & $8,40 \pm 4,82$ & $0,04^{*}$ \\
\hline & Duração do esforço (min) & $158,12 \pm 111,53$ & $154,23 \pm 109,02$ & $161,48 \pm 13,74$ & 0,46 \\
\hline \multirow[t]{2}{*}{ Somatório moderada e vigorosa } & Frequência semanal (dias) & $14,00 \pm 4,50$ & $4,10 \pm 3,60$ & $4,80 \pm 3,70$ & $0,03^{*}$ \\
\hline & Duração do esforço (min) & $420,00 \pm 105,40$ & $106,65 \pm 92,40$ & $104,33 \pm 90,30$ & 0,77 \\
\hline
\end{tabular}

GLM univariado; $\mathrm{p}<0,05^{\star}$

física, como ficar sentados diariamente em longo período. Os alunos das escolas de tempo integral afirmaram ficar mais tempo sentados em relação aos alunos das escolas de tempo parcial $(10,15 \pm 2,80$ versus $9,08 \pm 3,48$ horas, $\mathrm{p}<0,001)$ em dia da semana. Em dia de final de semana, os alunos das escolas de tempo integral também permanecem mais tempo sentados $(8,73 \pm 4,32$ versus $9,87 \pm 4,32$ horas, $\mathrm{p}=0,002)$. A média de comportamento sedentário dos alunos das escolas avaliadas foi de $18,78 \pm 6,23$ horas, sendo maior nas escolas de tempo integral $(20,03 \pm 5,51 \mathrm{ver}$ sus $17,81 \pm 6,53$ horas, $\mathrm{p}<0,001)$.

A partir do questionário EDAE-A, foi possível rastrear os sintomas de depressão, ansiedade e estresse dos participantes da pesquisa. Quanto ao estresse, dos 516 adolescentes, 99(19,2\%) responderam que têm dificuldade em se acalmar na maior parte da semana; $137(26,6 \%)$ tiveram reaçóes exageradas às situaçóes algumas vezes na semana; $130(25,2 \%)$ afirmaram que estavam bastante nervosos na maior parte da semana; $147(28,5 \%)$ disseram que algumas vezes na semana notaram que estavam ficando agitados e não tiveram paciência quando foram interrompidos; 133(25,8\%), durante algumas vezes na semana, acharam difícil relaxar, indicando sintomas de estresse.

Com relação ao transtorno de ansiedade, $170(32,9 \%)$ estudantes relataram experimentar boca seca algumas vezes na semana; 58(11,2\%) disseram ter dificuldade para respirar na maior parte da semana, bem como alguns tremores e preocupação de entrar em pânico.

Entretanto, quando questionados se estavam prestes a entrar em pânico, 43(18\%) estudantes de escola de tempo integral e $29(10,5 \%)$ estudantes de escola de tempo parcial afirmaram que acontecia al- gumas vezes na semana; 98(19\%) relataram que o coração estava mais acelerado sem ter feito esforço físico em algumas vezes da semana; e 106(20,5\%) afirmaram que se sentiam assustados sem ter motivo durante algumas vezes na semana.

Ao comparar os sintomas de ansiedade, estresse e depressão dos estudantes adolescentes de escolas de tempo parcial e integral, não se encontrou diferença, conforme demonstrado na tabela 3 .

Tabela 3. Comparação dos sintomas de ansiedade, estresse e depressão entre estudantes de tempo integral e parcial

\begin{tabular}{|c|c|c|c|c|}
\hline Domínios do EDAE-A & Grupos & $\mathrm{n}$ & Média \pm DP & $p$-value \\
\hline \multirow[t]{2}{*}{ Depressão } & Parcial & 277 & $7,05 \pm 5,84$ & \\
\hline & Integral & 239 & $7,56 \pm 5,81$ & 0,24 \\
\hline \multirow[t]{2}{*}{ Ansiedade } & Parcial & 277 & $5,21 \pm 4,74$ & \\
\hline & Integral & 239 & $5,87 \pm 5,05$ & 0,20 \\
\hline \multirow[t]{2}{*}{ Estresse } & Parcial & 277 & $8,50 \pm 6,00$ & \\
\hline & Integral & 239 & $9,11 \pm 5,60$ & 0,18 \\
\hline \multirow[t]{2}{*}{ Total (EDAE-A) } & Parcial & 277 & $20,77 \pm 14,84$ & \\
\hline & Integral & 239 & $22,55 \pm 14,50$ & 0,17 \\
\hline
\end{tabular}

GLM univariado, $p<0,05$; EDAE-A - Escala de Depressão, Ansiedade e Estresse em adolescentes.

Dos 516 participantes do estudo, 232(45\%) relataram que náo tiveram pensamentos positivos na semana; 54(29,8\%) relataram ter dificuldade de iniciativa para fazer as coisas durante algumas vezes na semana; 141(27,3\%) não tinham expectativa positiva em algumas vezes na semana; $132(25,6 \%)$ se sentiam abatidos e tristes na maior parte da semana; $106(20,5 \%)$ disseram que não tinham muito valor como pessoa na maior parte da semana, indicando sintomas de depressão. Quando comparados, houve uma maior proporção de "afetos negativos" de depressão nos alunos de escola de tempo integral $(p=0,03)$, pois os adolescentes relataram sentimentos de que a vida não tinha sentido durante boa parte da semana, conforme demonstrado na tabela 4 . 
Tabela 4. Comparação entre os grupos de tempo integral e parcial quanto aos sintomas de depressão, ansiedade e estresse

\begin{tabular}{|c|c|c|c|c|c|}
\hline $\begin{array}{l}\text { Afirmativa } \\
\text { (domínio) }\end{array}$ & Afirmativa ao aluno durante a semana & $\begin{array}{l}\text { Total } \\
\mathrm{n}(\%)\end{array}$ & $\begin{array}{c}\text { Período } \\
\text { integral } \\
\mathrm{n}(\%)\end{array}$ & $\begin{array}{c}\text { Período } \\
\text { parcial } \\
\mathrm{n}(\%)\end{array}$ & $p$-value \\
\hline \multirow{4}{*}{$\begin{array}{l}\text { Senti que estava prestes a } \\
\text { entrar em pânico } \\
\text { (ansiedade) }\end{array}$} & Não aconteceu na semana & $359(69,6)$ & $161(67,4)$ & $198(71,5)$ & \multirow[t]{4}{*}{0,05} \\
\hline & Aconteceu algumas vezes & $72(14)$ & $43(18)$ & $29(10,5)$ & \\
\hline & Aconteceu em boa parte da semana & $52(10,1)$ & $19(7,9)$ & $33(11,9)$ & \\
\hline & Aconteceu na maior parte da semana & $33(6,4)$ & $16(6,7)$ & $17(6,1)$ & \\
\hline \multirow{4}{*}{$\begin{array}{l}\text { Senti-me assustado sem ter } \\
\text { motivo } \\
\text { (ansiedade) }\end{array}$} & Não aconteceu na semana & $313(60,7)$ & $141(59)$ & $172(62,1)$ & \multirow[t]{4}{*}{0,06} \\
\hline & Aconteceu algumas vezes & $106(20,5)$ & $47(19,7)$ & $59(21,3)$ & \\
\hline & Aconteceu em boa parte da semana & $56(10,9)$ & $35(14,6)$ & $21(7,6)$ & \\
\hline & Aconteceu na maior parte da semana & $41(7,9)$ & $16(6,7)$ & $25(9)$ & \\
\hline \multirow{4}{*}{$\begin{array}{l}\text { Coração acelerado sem esforço } \\
\text { físico } \\
\text { (estresse) }\end{array}$} & Não aconteceu na semana & $270(52,3)$ & $117(49)$ & $153(55,2)$ & \multirow[t]{4}{*}{0,07} \\
\hline & Aconteceu algumas vezes & $98(19)$ & $45(18,8)$ & $53(19,1)$ & \\
\hline & Aconteceu em boa parte da semana & $75(14,5)$ & $33(13,8)$ & $42(15,2)$ & \\
\hline & Aconteceu na maior parte da semana & $73(14,1)$ & $04(18,4)$ & $29(10,5)$ & \\
\hline \multirow{4}{*}{$\begin{array}{l}\text { Eu me sentia abatido e triste } \\
\text { (depressão) }\end{array}$} & Não aconteceu na semana & $171(33,1)$ & $78(32.6)$ & $93(33,6)$ & \multirow[t]{4}{*}{0,58} \\
\hline & Aconteceu algumas vezes & $131(25,4)$ & $55(23)$ & $76(27,4)$ & \\
\hline & Aconteceu em boa parte da semana & $82(15,9)$ & $41(17,2)$ & $41(14,8)$ & \\
\hline & Aconteceu na maior parte da semana & $132(25,6)$ & $65(27,2)$ & $67(24,2)$ & \\
\hline \multirow{4}{*}{$\begin{array}{l}\text { Senti que a vida não tinha } \\
\text { sentido } \\
\text { (depressão) }\end{array}$} & Não aconteceu na semana & $286(55,4)$ & $129(54)$ & $157(56,7)$ & \multirow[t]{4}{*}{$0,03^{*}$} \\
\hline & Aconteceu algumas vezes & $85(16,5)$ & $33(13,8)$ & $52(18,8)$ & \\
\hline & Aconteceu em boa parte da semana & $49(9,3)$ & $31(13)$ & $17(6,1)$ & \\
\hline & Aconteceu na maior parte da semana & $97(18,8)$ & $46(19,2)$ & $51(18,4)$ & \\
\hline
\end{tabular}

Teste Exato de Fisher/Qui-Quadrado $p<0,05$

\section{Discussão}

Os resultados do presente estudo indicam que os estudantes adolescentes são inativos, sendo que nas escolas de tempo integral, a situação é ainda mais grave quando comparadas as de tempo parcial. A inatividade física no presente estudo foi maior que em outros que utilizaram o mesmo critério $(<300$ minutos por semana).Revisão sistemática que avaliou estudos internacionais identificou prevalências de 65,4 a $89,0 \% .{ }^{(15)}$ Estudos nacionais mostraram prevalências de $65,5 \%{ }^{(16)}$ e de $64,7 \%$ em meninos e $81,5 \%$ em meninas. ${ }^{(17)}$

Escolas de tempo integral originalmente tratam da ampliação da jornada escolar, expondo o adolescente a um maior período de atividades escolares, como teatro, dança e práticas esportivas no contra turno. ${ }^{(18)}$ Entretanto, quando analisamos os resultados deste estudo, percebemos que a suposiçáo de que o adolescente permanece mais tempo dentro da escola e, portanto, faria mais atividades físicas, não foi confirmada com os dados obtidos. Ao contrário, houve maior porcentagem $(98,3 \%)$ de estudantes adolescentes inativos nesta modalidade de ensino.

O mais alarmante é que o sedentarismo e o comportamento sedentário dos adolescentes são fatores de risco à saúde, predispondo-os a doenças, como a síndrome metabólica, doenças cardiovasculares, diabetes tipo 2, distúrbio do sono, ansiedade, depressão e estresse, dentre outras. ${ }^{(19)}$

Distúrbios apresentados durante a adolescência podem ocasionar prejuízo na idade adulta, com o aparecimento de doenças crônicas, como hipertensão arterial, obesidade e sedentarismo. Estudo feito com 963 pacientes adultos usuários do SUS em Bauru, SP, sendo $73,4 \%$ do sexo feminino, com a média de idade de 65 anos, observou que somente $1,2 \%$ dos indivíduos apresentaram comportamento ativo na infância, adolescência e idade adulta. Os indivíduos ativos em duas fases da vida somaram 5,4\%; em apenas uma fase, 29,2\%; e sedentários durante toda a vida,64,2\%. ${ }^{(20)}$ Estudo semelhante feito em 2010 com adultos usuários do SUS em Goiânia (GO) identificou que 59,8\% desta população apresentou baixos níveis de atividades físicas. ${ }^{(21)}$ As altas taxas de inatividade física em adolescentes são preocupantes, principalmente quando se sabe que esse tipo de comportamento tende a se perpetuar pelo resto da vida.

A frequência de comportamento sedentário foi maior que o recomendado tanto em estudantes de escolas de tempo parcial quanto de tempo integral, entretanto o último grupo apresentou maior tempo de comportamento sedentário que o primeiro tanto 
nos dias da semana quanto nos finais de semana, sugerindo comportamento sedentário talvez pelo uso de tela e/ou tarefas escolares. Estudo que avaliou mais de 100 mil escolares no Brasil identificou $68,15 \%$ de alunos comportamento sedentário (mais que duas horas por dia). ${ }^{(22)}$ É preocupante o fato de que poucos adolescentes estudantes se envolvam em atividades físicas, com isso, não cumprindo as recomendaçóes da OMS de, pelo menos, 60 minutos diários de atividade física moderada a vigorosa, principalmente quando são consideradas as evidências de que os hábitos da prática habitual de atividade física são importantes na prevenção de doenças crônicas não transmissíveis. ${ }^{(19)}$

$\mathrm{O}$ engajamento dos adolescentes estudantes em programas de atividade física deveria ir além das aulas de educação física, principalmente nas escolas de tempo integral, em que o aluno passa boa parte do dia dentro da instituição, com tarefas teóricas, sentados e de pouca movimentação na parte motora. Estudo nacional realizado utilizando o questionário PeNSE 2015, com 16.698 adolescentes, apontou que 69,2\% dos alunos de escolas públicas possuem quadra de esportes para a realização da prática de atividade física, sendo que $92 \%$ contam com a disponibilidade de materiais para a prática esportiva e que $22,2 \%$ dos alunos têm acesso a vestiários em condiçôes de uso. ${ }^{(23)}$

Nesse sentido, sugere-se que os estudantes adolescentes, principalmente das escolas de tempo integral, devam ter acesso à prática de atividade física de forma moderada a vigorosa por, pelo menos, 60 minutos diariamente, dentro ou fora das escolas e que essas informaçóes e experiências vindas, principalmente, das escolas, estimulem a possibilidade de atitude e independência diante à prática de atividade física no decorrer de toda sua vida. Estudos realizados em Maringá, com 64 adolescentes, e em Mandaguari, com 277 adolescentes, ambos do estado do Paraná, demonstraram a importância da motivação intrínseca dos estudantes através de projetos de extensão, principalmente o Projeto Segundo Tempo, que está presente nas escolas de tempo integral, estimulando a possibilidade da prática de atividade física dentro da escola, proporcionando lazer, melhora da aptidão física e aperfeiçoamento técnico em algum esporte. ${ }^{(24,25)}$
Entretanto, um estudo de revisão sistemática aponta o desinteresse dos adolescentes pela prática da atividade física após a aula. Mesmo as escolas sendo um cenário importante na promoção de atividade física e reduçáo dos comportamentos sedentários, as instalações precárias atuais existentes nas escolas e uso excessivo de aparelhos eletrônicos nas aulas práticas minam a motivação intrínseca da prática de atividade física desenvolvida pelos estudantes adolescentes no ambiente escolar. ${ }^{(26)}$

Além disso, grande parte dos estudantes adolescentes experimenta sintomas de depressão, ansiedade e estresse. Neste estudo, quando comparadas as características entre as escolas, houve uma maior proporção de estudantes da escola de tempo integral com sintomas negativos de depressáo. Estudos apontam resultados para o rastreamento dos episódios de depressão na adolescência semelhantes ao encontrado em nosso estudo. ${ }^{(27-29)}$

$\mathrm{O}$ adolescente estudante também vivencia, durante sua jornada escolar e adolescência, de acordo com o Diagnóstico Estatístico Mental (DSM-V), aspectos negativos de sintomas de ansiedade, com afirmativas de que sentiu que estava prestes a entrar em pânico,sentindo-se assustado sem ter motivo. ${ }^{(30)}$ Semelhante ao nosso resultado, estudo feito com 407 estudantes adolescentes de 14 a 18 anos na cidade de Maceió (AL) demonstrou que a maioria $(52 \%)$ tinha um grau de moderado a grave no aspecto negativo da ansiedade. ${ }^{(31)}$

Os adolescentes acabam passando boa parte de sua vida na escola, com convívio social necessário para sua formação acadêmica, porém o ambiente escolar apresenta algumas situaçóes de estresse potencial que podem agravar os sintomas negativos de ansiedade, como responder a perguntas em sala, apresentação oral e interação em grupos. ${ }^{(32)}$

Nesse cenário, os adolescentes podem sofrer bullying, serem ridicularizados e até mesmo vitimizados, parando de frequentar as aulas, distanciando-se de seus colegas e amizades, levando-os a profundos efeitos na saúde mental e reforçando a evasão escolar. ${ }^{(33)}$

Além de todo esse cenário no contexto escolar e da pouca atividade física desenvolvida por esses jovens, a tecnologia parece ser uma aliada aos sinto- 
mas negativos de ansiedade e agressividade. Estudo realizado em 2015, com 264 adolescentes escolares de Recife (PE), demonstrou que 99,6\% dos participantes têm acesso à internet e que o dispositivo tecnológico mais usado foi o smartphone $(65,2 \%)$, por um período acima de dez horas por dia, e que a proibição do uso dos dispositivos tecnológicos reforça os sentimentos de agressividade e irritabilidade, ocasionando sintomas de estresse. ${ }^{(34)}$

Em nosso estudo, os estudantes adolescentes apresentaram, de alguma forma, manifestaçôes de sintomas de aspectos negativos do estresse (72,1\%);dificuldade em se acalmar; reações exageradas às situaçóes;muito nervosos e agitados; sem paciência quando interrompidos; e dificuldade em relaxar.Estudo feito com adolescentes estudantes de Florianópolis (SC) demonstrou resultados semelhantes com $76,4 \%$ de estudantes que apresentavam a predominância de estresse psicológico. ${ }^{(35)}$ Outro estudo realizado em São Paulo, com 83 adolescentes escolares, identificou que $49,40 \%$ desses apresentaram transtornos de ansiedade/depressão, problemas de sociabilidade e comportamento agressivo. Além disso, 33,33\% apresentaram os sintomas negativos de estresse psicológico, cognitivo e fisiológico. ${ }^{(36)}$

$\mathrm{O}$ estudo apresenta limitaçóes, pois existem instrumentos e técnicas mais eficazes do que os questionários para avaliar a atividade física, tais como sensores eletrônicos de movimento para avaliação da atividade física habitual e identificadores fisiológicos, como também parâmetros bioquímicos para a detecção dos sintomas de depressão, ansiedade e estresse nos adolescentes ou mesmo a análise do profissional especializado. Contudo, esses métodos acabam inviabilizando as pesquisas populacionais pelo alto custo dos monitores e a logística complexa de colocação dos aparelhos, que envolve grande demanda de profissionais, além de conhecimentos técnicos específicos, seja com o modelo, ou software, ou análise dos dados.

Questionários costumam supervalorizar a prática da atividade física quando comparados a outros procedimentos de medição eletrônicos e mecânicos, como monitores de frequência cardíaca, pedômetros e acelerômetros. ${ }^{(37)}$ Entretanto, os questionários são menos onerosos e fáceis de manusear e gerir, sendo vantajosos em amplos estudos epidemiológicos. ${ }^{(38)}$
Outros estudos são imprescindíveis para definir se os aspectos negativos de depressão, ansiedade e estresse estão associados ao nível de atividade física em estudantes adolescentes, visto que a coexistência desses fatores torna os alunos propensos a doenças no presente e no futuro. Ressalta-seque as escolas e a família são formidáveis pilares em semelhança aos hábitos de vida saudáveis, por isso, influências nessas esferas podem ser ideais para a promoção de saúde e adequada condição de vida.

\section{Conclusão}

Os estudantes adolescentes do sistema público de ensino demonstraram alto índice de inatividade físi$\mathrm{ca}$, não atendendo às recomendaçóes da OMS, com maior proporção de inativos nas escolas de período integral. Além disso, esses estudantes vivenciam "afetos negativos" de ansiedade, estresse e depressão. Acreditamos que este estudo colaborou com achados importantes, e é um alerta para os sistemas públicos de educação e saúde, especialmente para as escolas de tempo integral, pois podem fundamentar políticas públicas de incentivo à prática de atividade física para adolescentes nas escolas estaduais; educação social sobre os benefícios fisiológicos dos exercícios na prevenção de doenças crônicas, transtornos mentais; e conscientização dos estudantes adolescentes sobre a realização e importância de atividades físicas durante a adolescência e na idade adulta.

\section{Colaborações}

Costa MPS, Schmidt A, Vitorino PVO e Corrêa KS declaram que contribuíram com a concepção do estudo, análise e interpretação dos dados, redação do artigo, revisão crítica relevante do conteúdo intelectual e aprovação da versão final a ser publicada.

\section{Referências}

1. World Health Organization (WHO). World health statistics 2010. Geneva: WHO; 2010. 
2. Rothon C, Edwards P, Bhui K, Viner RM, Taylor S, Stansfeld SA. Physical activity and depressive symptoms in adolescents: a prospective study. BMC Med. 2010;8(1):32.

3. Martins RC, Ricardo LI, Mendonça G, de Rosa DL, da Gama Bastos $\mathrm{LL}$, de Vargas Nunes Coll C, et al. Temporal trends of physical activity and sedentary behavior simultaneity in brazilian students. J Phys Act Health. 2018;15(5):331-7.

4. World Health Organization (WHO). Physical activity. Geneva: WHO; 2018.

5. Arundell L, Fletcher E, Salmon J, Veitch J, Hinkley T. A systematic review of the prevalence of sedentary behavior during the after-school period among children aged 5-18 years. Int J Behav Nutr Phys Act. 2016;13(1):93.

6. Guedes DP, Souza MV, Ferreirinha JE, Silva AJ. Physical activity and determinants of sedentary behavior in Brazilian adolescents from an underdeveloped region. Percept Mot Skills. 2012;114(2):542-52.

7. Vitorino PV, Barbosa MA, Sousa AL, Jardim PC, Ferreira SS. Prevalência de estilo de vida sedentário entre adolescentes. Acta Paul Enferm. 2015;28(2):166-71.

8. Patias ND, Machado WD, Bandeira DR, Dell'Aglio DD, et al. Depression Anxiety and Stress Scale (DASS-21)-Short Form: Adaptation and Validation for Brazilian Adolescents. Psico-USF. 2016;21(3):459-69.

9. Liu M, Wu L, Yao S. Dose-response association of screen time-based sedentary behavior in children and adolescents and depression: a metaanalysis of observational studies. Br J Sports Med. 2016;50(20):1252-8.

10. Schuch FB, Vancampfort D, Firth J, Rosenbaum S, Ward PB, Silva ES, et al. Physical activity and incident depression: a meta-analysis of prospective cohort studies. Am J Psychiatry. 2018;175(7):631-48.

11. Stubbs B, Koyanagi A, Hallgren M, Firth J, Richards J, Schuch F, et al. Physical activity and anxiety: A perspective from the World Health Survey. J Affect Disord. 2017;208:545-52.

12. Stubbs B, Vancampfort D, Rosenbaum S, Firth J, Cosco T, Veronese N, et al. An examination of the anxiolytic effects of exercise for people with anxiety and stress-related disorders: A meta-analysis. Psychiatry Res. 2017;249:102-8.

13. Guedes DP, Lopes CC, Guedes JE. Reprodutibilidade e validade do Questionário Internacional de Atividade Física em adolescentes. Rev Bras Med Esporte. 2005;11(2):151-8.

14. Tremblay MS, LeBlanc AG, Kho ME, Saunders TJ, Larouche R, Colley $\mathrm{RC}$, et al. Systematic review of sedentary behaviour and health indicators in school-aged children and youth. Int J Behav Nutr Phys Act. 2011;8(1):98.

15. Sharara E, Akik C, Ghattas H, Makhlouf Obermeyer C. Physical inactivity, gender and culture in Arab countries: a systematic assessment of the literature. BMC Public Health. 2018;18(1):639.

16. Werneck AO, Oyeyemi AL, Fernandes RA, Romanzini M, Ronque ER, Cyrino ES, et al. Regional socioeconomic inequalities in physical activity and sedentary behavior among brazilian adolescents. J Phys Act Health. 2018;15(5):338-44.

17. Werneck AO, Collings PJ, Barboza LL, Stubbs B, Silva DR. Associations of sedentary behaviors and physical activity with social isolation in 100,839 school students: The Brazilian Scholar Health Survey. Gen Hosp Psychiatry. 2019;59:7-13.

18. Vasconcelos RD. As políticas públicas de educação integral, a escola unitária e a formação onilateral [tese]. Brasília (DF): Universidade de Brasília; 2012

19. Alvarez-Pitti J, Casajús Mallén JA, Leis Trabazo R, Lucía A, López de Lara D, Moreno Aznar LA, et al. [Exercise as medicine in chronic diseases during childhood and adolescence]. An Pediatr (Barc). 2020;92(3):173.e1-8. Spanish.
20. Turi BC, Codogno JS, Fernandes RA, Monteiro HL, et al. Prática de atividade física, adiposidade corporal e hipertensão em usuários do Sistema Único de Saúde. Rev Bras Epidemiol. 2014;17(4):925-37.

21. Ferreira CCDC, Peixoto MDRG, Barbosa MA, Silveira EAD, et al. Prevalência de fatores de risco cardiovascular em idosos usuários do Sistema Único de Saúde de Goiânia. Arq Bras Cardiol. 2010; 95(5): 621-8.

22. Silva RM, Andrade AC, Caiaffa WT, Medeiros DS, Bezerra VM. National Adolescent School-based Health Survey - PeNSE 2015: sedentary behavior and its correlates. PLoS One. 2020;15(1):e0228373.

23. Instituto Brasileiro de Geografia e Estatística (IBGE). Pesquisa nacional de saúde escolar. Rio de Janeiro: IBGE; 2016.

24. Oliveira AA, Teixeira CA, Rechenchosky L, Oliveira Filho A, Prati AC, Costa LA, et al. Motivação para a prática esportiva: Programa Segundo Tempo, Região 16. Rev Digital (Buenos Aires). 2010;15(150): 1-11.

25. Polles MC, Barbosa CC. Motivação de adolescentes de 10 a 18 anos em participar de projetos que envolvam a prática de atividades físicas. Diálogos \& Saberes. 2012; 8(1):247-65.

26. Morton KL, Atkin AJ, Corder K, Suhrcke M, van Sluijs EM. The school environment and adolescent physical activity and sedentary behaviour: a mixed-studies systematic review. Obes Rev. 2016;17(2):142-58.

27. Salle E, Rocha NS, Rocha TS, Nunes C, Chaves ML. Escalas psicométricas como instrumentos de rastreamento para depressão em estudantes do ensino médio. Arch Clin Psychiatry. 2012;39(1):24-7.

28. Kessler RC, Petukhova M, Sampson NA, Zaslavsky AM, Wittchen $\mathrm{HU}$. Twelve-month and lifetime prevalence and lifetime morbid risk of anxiety and mood disorders in the United States. Int J Methods Psychiatr Res. 2012;21(3):169-84.

29. Kessler RC, Avenevoli S, Costello EJ, Georgiades K, Green JG, Gruber $\mathrm{MJ}$, et al. Prevalence, persistence, and sociodemographic correlates of DSM-IV disorders in the National Comorbidity Survey Replication Adolescent Supplement. Arch Gen Psychiatry. 2012;69(4):372-80.

30. American Psychiatry Association (APA). Diagnostic and Statistical Manual of Mental Disorders (DSM-5) 5th ed. Washington, DC: APA; 2013.

31. Lopes AP, Rezende MM. Anxiety and the use of psychoactive substances among adolescents. Estud Psicol (Campinas). 2013;30(1):49-56.

32. Blöte AW, Miers AC, Heyne DA, Westenberg PM, et al. Social anxiety and the school environment of adolescents. Social anxiety and phobia in adolescents. Cham: Springer; 2015. p. 151-81.

33. Schermann LB, Béria JU, Jacob MH, Arossi G, Benchaya MC, Bisch NK, et al. Estresse em adolescentes: estudo com escolares de uma cidade do sul do Brasil. Aletheia. 2014;(43/44):170-83.

34. Silva RA, Silva PM, Moura JF, Santos DC, Raposo JC, Gomes BD, et al. Adolescentes e abuso de tecnologias: um indicativo de problemas comportamentais? Adolesc Saude. 2017;14(3):77-82.

35. Pires EA, Duarte MD, Pires MC, Sá e Souza G. Hábito de atividade física e 0 estresse em adolescentes de Florianópolis. Rev Bras Ciênc Mov. 2008;12(1):51-6.

36. Justo AP, Enumo SR. Problemas emocionais e de comportamento na adolescência: 0 papel do estresse. Bol Acad Paul Psicol. 2015;35(89):350-70.

37. Shephard RJ. Limits to the measurement of habitual physical activity by questionnaires. Br J Sports Med. 2003;37(3):197-206.

38. Sallis JF, Saelens BE. Assessment of physical activity by selfreport: status, limitations, and future directions. Res Q Exerc Sport. 2000;71(Suppl 2):1-14. 